\title{
Walking signals in eight-flavor QCD on the lattice
}

Yasumichi Aoki $^{a}$, Tatsumi Aoyama $^{a}$, Masafumi Kurachi ${ }^{a}$, Toshihide Maskawa $^{a}$, Kohtaroh Miura $^{a}$, Kei-ichi Nagai $^{* \dagger a}$, Hiroshi Ohki $^{a}$, Enrico Rinaldi ${ }^{b}$, Akihiro Shibata ${ }^{c}$, Koichi Yamawaki $^{a}$, Takeshi Yamazaki $\stackrel{\ddagger a}{ }$

\section{(LatKMI Collaboration)}

${ }^{a}$ Kobayashi-Maskawa Institute for the Origin of Particles and the Universe (KMI), Nagoya University, Nagoya, 464-8602, Japan

${ }^{b}$ Lawrence Livermore National Laboratory, Livermore, California 94550, USA

${ }^{c}$ Computing Research Center, High Energy Accelerator Research Organization (KEK),

Tsukuba, 305-0801, Japan

\begin{abstract}
We investigate walking signals of $N_{f}=8$ QCD through the meson spectrum, using the HISQ action. Our data for $N_{f}=8 \mathrm{QCD}$ are consistent with chiral perturbation theory (ChPT) in their chiral extrapolations, hence with the theory exhibiting spontaneous chiral symmetry breaking. Remarkably, while the $N_{f}=8$ data near the chiral limit are well described by the ChPT, those for the relatively large fermion bare mass $m_{f}$ away from the chiral limit actually exhibit a finite-size hyperscaling relation, suggesting a large anomalous dimension $\gamma_{m} \sim 1$. This implies that there exists a remnant of the infrared conformality, and suggests that a typical "one-family" technicolor model, as modeled by $N_{f}=8 \mathrm{QCD}$, can be a walking technicolor theory.
\end{abstract}

KMI International Symposium 2013 on" Quest for the Origin of Particles and the Universe", 11-13 December, 2013

Nagoya University, Japan

\footnotetext{
* Speaker.

${ }^{\dagger}$ E-mail: keiichi.nagai@kmi.nagoya-u.ac.jp

¥Present address: Graduate School of Pure and Applied Sciences, University of Tsukuba, 305-8571, Japan
} 


\section{Introduction}

One of the candidates for the theory beyond the SM is Walking Technicolor (WTC) [U]. It suggests an approximate scale invariance with a large anomalous dimension $\gamma_{m} \simeq 1$ due to the "walking" coupling (Fig. (W), which is based on the scale-invariant gauge dynamics []]. The walking behavior can in fact be realized in "large $N_{f}$ QCD". $N_{f}=8$ is particularly interesting from the model-building point of view: it is none other than so-called "one-family model" (Farhi-Susskind model [ [ $]$ ] ). Thus if the $N_{f}=8$ turns out to be a walking theory, it would be a great message for the phenomenology, which is to be tested by the on-going experiments at LHC. (See Ref. [四] and references therein for the review of the recent lattice studies.)

As in Fig. I, the fermion bare mass $m_{f}$ obviously distorts the ideal behavior of the breaking of the scale symmetry. (This is in contrast to the conformal case, e.g. our $N_{f}=12$ result [[]].) Then, disregarding the effects of the lattice parameters $L$ and $a$ for the moment ${ }^{1}$, we may imagine possible effects of $m_{f}$ on the walking coupling of our target of study. Indeed, in our numerical exploration with $N_{f}=8$ [四], while the data near the chiral limit are well described by the ChPT, those for the relatively large $m_{f}$ away from the chiral limit actually exhibit a finite-size hyperscaling (FSHS) relation, suggesting a large anomalous dimension $\gamma_{m} \sim 1$. This is the first time that such a relation is observed in a theory with spontaneous chiral symmetry breaking ${ }^{2}(\mathrm{~S} \chi \mathrm{SB})$.
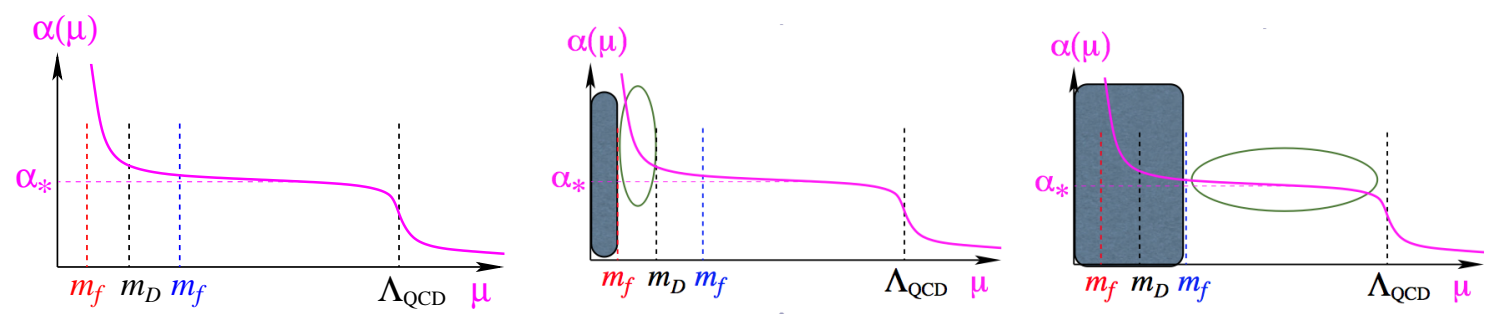

Figure 1: Schematic two-loop/ladder picture of the gauge coupling of the massless large- $N_{f}$ QCD as a walking gauge theory in the $\mathrm{S} \chi \mathrm{SB}$ phase near the conformal window (left). $m_{D}$ is the dynamical mass of the fermion generated by the S $\chi \mathrm{SB}$. Case 1: $m_{f} \ll m_{D}$ (red) well described by ChPT (center), and Case 2: $m_{f} \gg m_{D}$ (blue) well described by the hyperscaling (right). The S $\chi \mathrm{SB}$ order parameter on the lattice is not $m_{D}$ but would be the chiral limit of $F_{\pi}, F=F_{\pi}\left(m_{f}=0\right)$, which would be expected roughly as $m_{D}=\mathscr{O}(F)$.

\section{Lattice simulation and results}

In our simulation, we use the tree-level Symanzik/HISQ action [四] (without tadpole improvement and the mass correction in the Naik term) for the improvement of the flavor symmetry and the behavior towards the continuum limit. We carry out the simulation by using the standard Hybrid Monte-Carlo (HMC) algorithm and measure the mass of the pion $M_{\pi}, \rho$-meson $M_{\rho}$, the decay constant of the pion $F_{\pi}$ and the chiral condensate $\langle\bar{\psi} \psi\rangle$ as the basic observables, for various quark

\footnotetext{
${ }^{1}$ In our simulation we use the parameter region where the effect of the system size is subdominant compared to the mass effect. This strategy is different from the one which is advocated by the authors of Ref. [G].

${ }^{2}$ Ref. [ $\left[\right.$ ] found that $N_{f}=8 \mathrm{QCD}$ with domain-wall fermions has $\mathrm{S} \chi \mathrm{SB}$ and conformal properties depending on the fermion mass region.
} 
masses and on various lattices, $L^{3} \times T$ with fixed aspect ratio $T / L=4 / 3$ for $L=12,18,24,30$ and 36 at $\beta=3.8$.

We performed the analysis based on ChPT and FSHS. If the simulation region of $m_{f}$ is in the $\mathrm{S} \chi \mathrm{SB}$ region, physical quantities in the spectroscopy, $M_{H}$ for $H=\pi, \rho, \cdots$ and $F_{\pi}$, are described by the ChPT. In that case, the masses and $F_{\pi}$ depend on $m_{f}$ up to chiral $\log$ as $M_{\pi}^{2}=C_{1}^{\pi} m_{f}+$ $C_{2}^{\pi} m_{f}^{2}+\cdots, \quad F_{\pi}=F+C_{1}^{F} m_{f}+C_{2}^{F} m_{f}^{2}+\cdots$, where $F$ is the value in the chiral limit. On the other hand, if the theory is in the conformal window, $M_{H}$ and $F_{\pi}$ in the infinite volume limit are described by the HS relation $M_{H} \propto m_{f}^{1 /\left(1+\gamma_{*}\right)}$, and on the finite volume are described by the FSHS $\xi_{H}=L M_{H}=\mathscr{F}_{H}\left(L m_{f}^{1 /\left(1+\gamma_{*}\right)}\right)$ for $H=\pi, \rho$ or $F\left(M_{H}=F_{\pi}\right)$. The function, $\mathscr{F}_{H}$, is a some function (unknown a priori) of the scaling variable $X=L m_{f}^{1 /\left(1+\gamma_{*}\right)}$ in which $\gamma_{*}$ denotes the mass anomalous dimension $\gamma_{m}$ at the infrared fixed point and its value is universal for all channels.

\section{Chiral perturbation theory (ChPT) analysis of $F_{\pi}, M_{\rho}$ and $\langle\bar{\psi} \psi\rangle$ in small $m_{f}$}

Based on the scenario explained in Fig. [, and the blowup behaviors of both $F_{\pi} / M_{\pi}$ (see Fig. [) and $M_{\rho} / M_{\pi}$ towards the chiral limit [Q], [8], we first attempt the ChPT analysis in the small $m_{f}$.
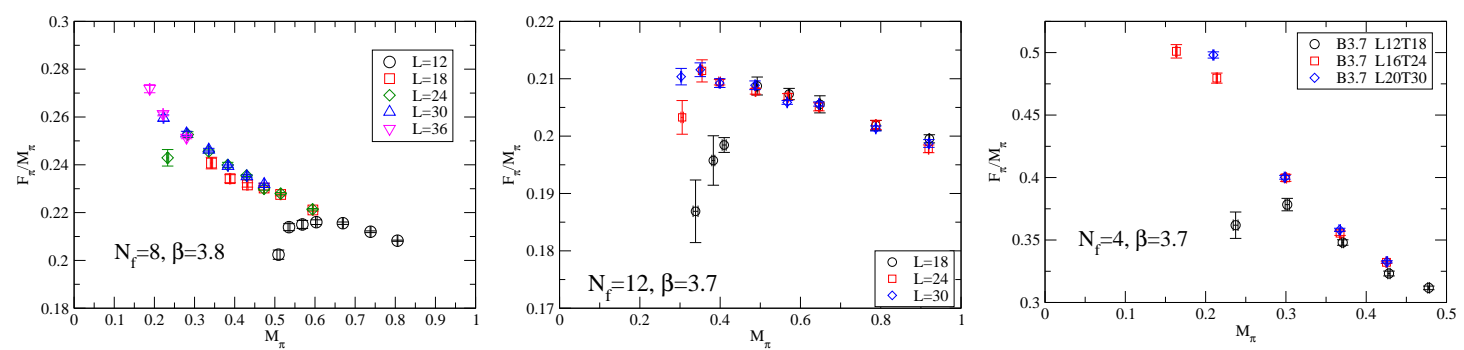

Figure 2: $F_{\pi} / M_{\pi}$ as a function of $M_{\pi}$ for $N_{f}=8$ (left), $N_{f}=12$ (center), and $N_{f}=4$ (right).

Table $\mathbb{W}$ shows the results of the quadratic fit of $F_{\pi}$. (The fitted data are the result on the largest volume at each $m_{f}$ region. ) Particularly for the small region, $0.015 \leq m_{f} \leq 0.04$, the polynomial fit gives a good $\chi^{2} /$ dof $(=0.46)$. When we include the data at $m_{f}=0.05, \chi^{2} /$ dof jumps up; this jump might be caused by the instability due to small dof. This suggests that there is a bound beyond which the ChPT does not describe the data well, and that bound is around $m_{f} \lesssim 0.05$. With this consideration and the good chiral behavior observed for other quantities (for instance $M_{\rho}$ and $\langle\bar{\psi} \psi\rangle$ ) for small $m_{f}$ region, we choose $m_{f}=0.015-0.04$ for the fitting range of all quantities (roughly corresponding to Case 1 in Fig. W). The above analysis suggests that our result in $N_{f}=8$ is consistent with the $\mathrm{S} \chi \mathrm{SB}$ phase with $F=0.0310(13)$ up to chiral log corrections. Figure B shows the ChPT fit ${ }^{3}$ in the range $0.015 \leq m_{f} \leq 0.04$ and the HS fit in the range $0.05 \leq m_{f}$. Since the behavior in the small $m_{f}$ region is well-described by the ChPT, therefore $N_{f}=8 \mathrm{QCD}$ is consistent with $\mathrm{S} \chi \mathrm{SB}$.

We next analyze whether the chiral condensate shows $\mathrm{S} \chi \mathrm{SB}$ behavior. We perform a direct measurement $\langle\bar{\psi} \psi\rangle=\operatorname{Tr}\left[D_{H I S Q}^{-1}(x, x)\right] / 4$, and the quantity $\Sigma \equiv \frac{F_{\pi}^{2} M_{\pi}^{2}}{4 m_{f}}$ through the GMOR relation.

\footnotetext{
${ }^{3}$ The left panel of Fig. [1] also shows the $L=12$ data of $F_{\pi}$, denoted as "region-A" in which $F_{\pi}$ linearly goes to zero towards the chiral limit. We therefore do not include such data in the analysis.
} 


\begin{tabular}{cccccc}
\hline fit range $\left(m_{f}\right)$ & $F$ & $\mathscr{X}\left(m_{f}^{\min }=0.015\right)$ & $\mathscr{X}\left(m_{f}=m_{\max }\right)$ & $\chi^{2} /$ dof & dof \\
\hline $0.015-0.04$ & $0.0310(13)$ & 3.74 & 11.80 & 0.46 & 1 \\
$0.015-0.05$ & $0.0278(8)$ & 4.64 & 19.28 & 5.56 & 2 \\
$0.015-0.10$ & $0.0311(3)$ & 3.70 & 37.0 & 7.85 & 6 \\
\hline
\end{tabular}

Table 1: Results of the chiral fit of $F_{\pi}$ with $F_{\pi}=F+C_{1}^{F} m_{f}+C_{2}^{F} m_{f}^{2}$ and the the expansion parameter in ChPT, $\mathscr{X}=N_{f}\left(\frac{M_{\pi}}{4 \pi F / \sqrt{2}}\right)^{2}$, for various fit ranges. This is in contrast to the $N_{f}=12$ case [[]] showing $\mathscr{X} \simeq 40$ at $m_{f}^{\min }$.
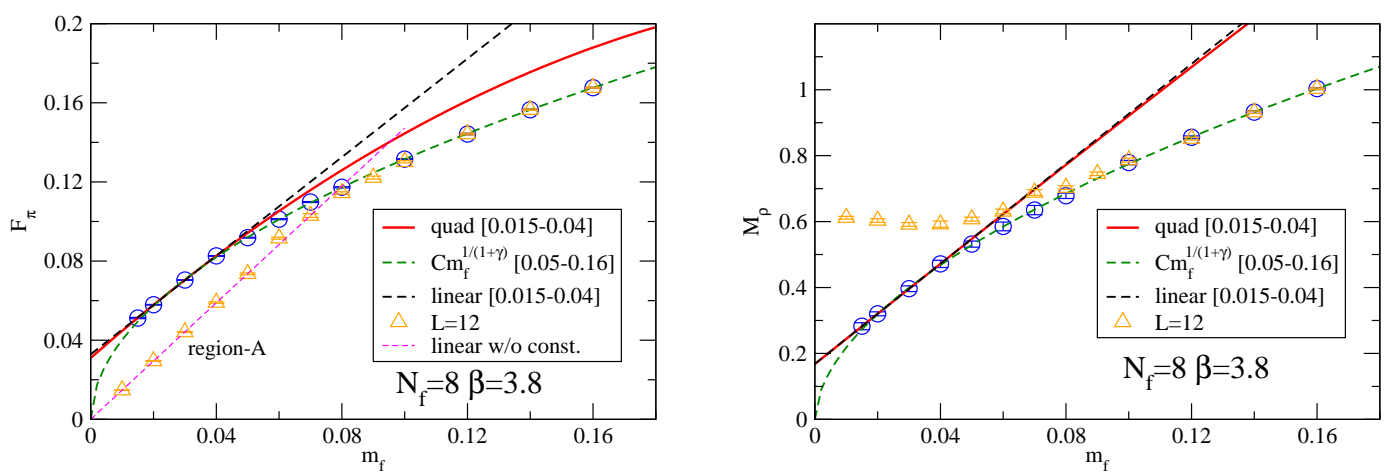

Figure 3: Fitting results of $F_{\pi}$ (left) and $M_{\rho}$ (right). Linear and quadratic fits in $0.015 \leq m_{f} \leq 0.04$, Power fit $\left(y=C m_{f}^{\alpha}\right)$ in $0.05 \leq m_{f} \leq 0.16$.

We also estimate the chiral limit, $\left.F^{2} \cdot\left(\frac{M_{\pi}^{2}}{4 m_{f}}\right)\right|_{m_{f} \rightarrow 0}=0.00050(3)$, by multiplying $F$ with the extrapolated value of $M_{\pi}^{2} / m_{f}$, as the alternative of the chiral limit. All the results in the chiral limit are non-zero and are consistent with one another.

From the analyses up to chiral $\log$ of all the observables, $F_{\pi}, M_{\pi}, M_{\rho}$ and $\langle\bar{\psi} \psi\rangle$, the chiral behavior of $N_{f}=8 \mathrm{QCD}$ is consistent with that of $\mathrm{S} \chi \mathrm{SB}$ in $0.015 \leq m_{f} \leq 0.04$.

\section{Study of remnants of conformality, Hyperscaling analysis in the intermediate $m_{f}$}

We find the two regions of $m_{f}$ having qualitatively different properties: $0.015 \leq m_{f} \leq 0.04$ and $0.05 \leq m_{f} \leq 0.16$ by the analysis of ChPT shown in the previous section. Furthermore, based on the scenario of WTC in Fig. W, if this theory is near the conformal phase boundary, it is expected that some remnants of the conformality appear in physical quantities. It is remarkable that in Fig. [] the fit results in the mass range, $m_{f} \gtrsim 0.05$, are consistent with the HS behavior. This suggests that, although $N_{f}=8 \mathrm{QCD}$ is in the $\mathrm{S} \chi \mathrm{SB}$ phase, there exists a remnant of the conformality in the $m_{f}$ region away from the chiral limit. Therefore, we will carry out further in depth analysis, which employs the FSHS test with the mass correction term [Q] , to investigate whether the remnant of the conformality persists.

\subsection{Finite size Hyper-Scaling (FSHS) fits with the correction term}

Our data of $N_{f}=8$ cannot satisfy the FSHS with universal $\gamma$ in the whole range of $m_{f}$ [ $[$ ], 
because we showed that the theory is in the $\mathrm{S} \chi \mathrm{SB}$ phase ${ }^{4}$. However, because of the power behavior in the middle range of the fermion mass as mentioned in Fig. B, we carry out the FSHS test with the mass correction term [Q] in our data to look for a remnant of the conformality.

Although it might be possible to obtain a common value of the $\gamma$, FSHS is only expected for larger mass region, where mass corrections may not be negligible [Q] , $\xi_{H}=C_{0}^{H}+C_{1}^{H} X+C_{2}^{H} L m_{f}^{\alpha}$ for $m_{f} \geq 0.05$ and $\xi_{\pi} \geq 8$. We perform a simultaneous fit with mass correction term [ $[$ ] in this region $^{5}$ of $m_{f}$ and $\xi_{\pi}$ using $M_{\pi}, F_{\pi}$, and $M_{\rho}$ with a common $\gamma$. The example with $\alpha=1$ of this analysis is shown in Fig. 因, as a typical result of the simultaneous fit. Under the assumption that all the observables give a universal $\gamma$, we estimate $\gamma=0.78-0.93$ with $\chi^{2} /$ dof $=O(0.1)$. These estimated values of $\gamma$ would be identified as the mass anomalous dimension in the walking regime.
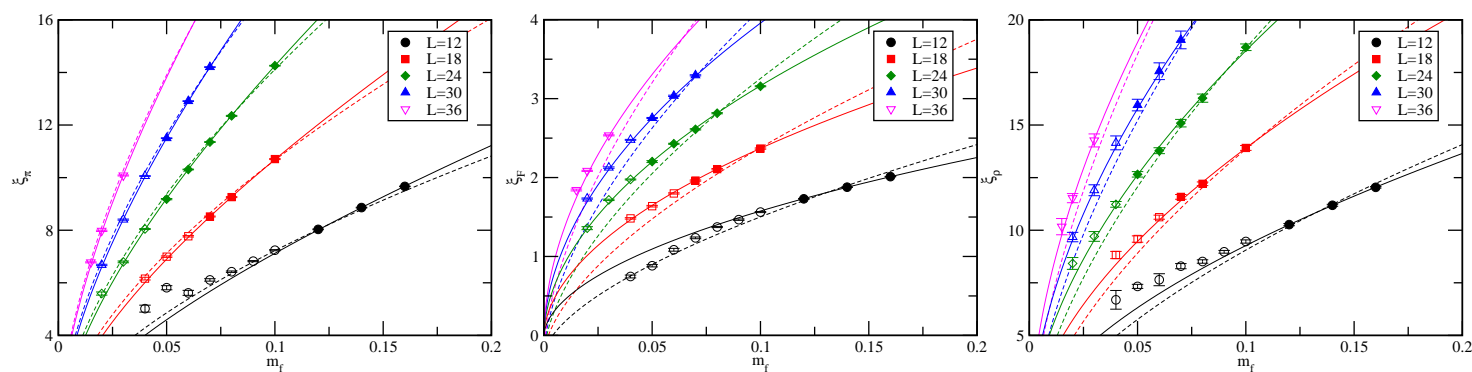

Figure 4: Simultaneous FSHS fit in $\xi_{\pi}$ (left), $\xi_{F}$ (center) and $\xi_{\rho}$ (right) with $\alpha=1$. The filled symbols are included in the fit, but the open symbols are omitted. The fitted region is $m_{f} \geq 0.05$ and $\xi_{\pi} \geq 8$. The solid curve is the fit result. For a comparison, the simultaneous fit result without correction terms is also plotted by the dashed curve, whose $\chi^{2} /$ dof $=83$.

\section{Summary and Discussion}

In search of a candidate for WTC theory, we have investigated the meson spectrum of $N_{f}=8$ QCD using lattice simulations based on the HISQ action for $\beta=6 / g^{2}=3.8$ [प]. We have found that the data of $F_{\pi}, M_{\pi}, M_{\rho}$ and $\langle\bar{\psi} \psi\rangle$ are well described by the ChPT in the small $m_{f}$ region, $0.015 \leq$ $m_{f} \leq 0.04$ (Case 1 in Fig. 四), suggesting that $F=0.031(1)\left({ }_{-10}^{+2}\right),\left.\langle\bar{\psi} \psi\rangle\right|_{m_{f} \rightarrow 0}=0.00052(5)\left({ }_{-29}^{+8}\right)$, and $\frac{M_{\rho}}{F / \sqrt{2}}=7.7(1.5)\left({ }_{-0.4}^{+3.8}\right)$ in the chiral limit extrapolation. In contrast, the data for the relatively large fermion bare mass $m_{f} \geq 0.05$, away from the chiral limit, actually exhibited a naive FSHS with the non-universal $\gamma$. This implies that there exists a remnant of the IR conformality where the $\mathrm{S} \chi \mathrm{SB}$ effects are negligible (Case 2 in Fig. W). Therefore, there could exist large mass corrections on the FSHS and we obtained $0.78 \lesssim \gamma \lesssim 0.93$ in simultaneous FSHS fits with mass correction for $m_{f} \geq 0.05$ and $\xi_{\pi} \geq 8$. Summarizing all our analyses we may infer that a typical technicolor

\footnotetext{
${ }^{4}$ The exponents for $F_{\pi}, M_{\rho}$ and $M_{\pi}$ obtained from naive FSHS relation are $\gamma=0.928(8), 0.798(20)$ and $0.567(3)$ respectively, being non-universal value. This non-universal value of $\gamma$ is also shown in Ref. [ [8].

${ }^{5}$ It is noted that a simultaneous fit including the lighter mass with $m_{f} \geq 0.015$ in $\xi_{\pi} \geq 6.8$ fails with a large $\chi^{2} /$ dof $=$ 3.5 even if the mass correction is included. This is because the chiral properties are dictated by $\mathrm{S} \chi \mathrm{SB}$ and should not be consistent with HS near the chiral limit.
} 
("one-family model") as modeled by $N_{f}=8$ QCD can be a WTC theory having an approximate scale invariance with large anomalous dimension $\gamma_{m} \sim 1$.

Finally, we should comment on the possible light flavor-singlet scalar meson in $N_{f}=8 \mathrm{QCD}$. The WTC predicts a light composite Higgs-like scalar boson (techni-dilaton) as a pseudo NambuGoldstone boson of the approximate scale invariance. We studied [U] both the flavor-singlet scalar and scalar glueballs in $N_{f}=12$ for a hint of a scalar bound state; we then studied the flavor-singlet scalar in $N_{f}=8$ and found it as light as $\pi$. The result suggests that a light flavor-singlet scalar composite does exist [प] in WTC theories.

\section{Acknowledgments}

We thank Ed Bennett for his careful reading of this manuscript. Numerical calculations have been carried out on the high-performance computing system $\varphi$ at KMI, Nagoya University, and the computer facilities of the Research Institute for Information Technology in Kyushu University. This work is supported by the JSPS Grant-in-Aid for Scientific Research (S) No.22224003, (C) No.23540300 (K.Y.), for Young Scientists (B) No.25800139 (H.O.) and No.25800138 (T.Y.), and also by Grants-in-Aid of the Japanese Ministry for Scientific Research on Innovative Areas No.23105708 (T.Y.). E.R. was supported by a SUPA Prize Studentship and a FY2012 JSPS Postdoctoral Fellowship for Foreign Researchers (short-term).

\section{References}

[1] K. Yamawaki, M. Bando, and K.-i. Matumoto, Phys. Rev. Lett. 56 (1986) 1335.

[2] T. Maskawa and H. Nakajima, Prog. Theor. Phys. 52 (1974) 1326-1354.

[3] E. Farhi and L. Susskind, Phys.Rev. D20 (1979) 3404-3411.

[4] J. Kuti, PoS LATTICE2013 (2013) 004.

[5] LatKMI Collaboration, Y. Aoki, T. Aoyama, M. Kurachi, T. Maskawa, K.-i. Nagai, H. Ohki, A. Shibata, K. Yamawaki, and T. Yamazaki, Phys. Rev. D 86 (2012) 054506.

[6] K.-I. Ishikawa, Y. Iwasaki, Y. Nakayama, and T. Yoshie, Phys. Rev. D 87 (2013) 071503.

[7] LatKMI Collaboration, Y. Aoki, T. Aoyama, M. Kurachi, T. Maskawa, K.-i. Nagai, H. Ohki, A. Shibata, K. Yamawaki, and T. Yamazaki, Phys. Rev. D 87 (2013) 094511.

[8] LSD Collaboration, T. Appelquist et. al.,

[9] LatKMI Collaboration, Y. Aoki, T. Aoyama, M. Kurachi, T. Maskawa, K.-i. Nagai, H. Ohki, A. Shibata, K. Yamawaki, and T. Yamazaki, Phys. Rev. D 85 (2012) 074502.

[10] LatKMI Collaboration, Y. Aoki, T. Aoyama, M. Kurachi, T. Maskawa, K.-i. Nagai, H. Ohki, E. Rinaldi, A. Shibata, K. Yamawaki, and T. Yamazaki, Phys.Rev.Lett. 111 (2013) 162001.

[11] LatKMI Collaboration, Y. Aoki, T. Aoyama, M. Kurachi, T. Maskawa, K. Miura, K.-i. Nagai, H. Ohki, E. Rinaldi, A. Shibata, K. Yamawaki, and T. Yamazaki, Phys.Rev. D89 (2014) 111502(R). 